\title{
Long-term cortisol levels are not associated with nasal carriage of Staphylococcus aureus
}

\author{
L. Manenschijn • A. M. Jetten • W. J. B. van Wamel • \\ M. Tavakol • J. W. Koper • E. L. T. van den Akker • \\ A. van Belkum • E. F. C. van Rossum
}

Received: 11 April 2011 / Accepted: 18 April 2011 /Published online: 15 May 2011

(C) The Author(s) 2011. This article is published with open access at Springerlink.com

\begin{abstract}
Staphylococcus aureus (S. aureus) colonizes the anterior nares in part of the population and the persistent carrier state is associated with increased infection risk. Knowledge concerning the determinants of $S$. aureus nasal carriage is limited. Previously, we found that glucocorticoid receptor polymorphisms influence carrier risk, suggesting involvement of glucocorticoids. Our aim was to study long-term cortisol levels in non-carriers, intermittent, and persistent carriers of $S$. aureus. We hypothesized that cortisol levels are higher in carriers, since cortisol-induced immune suppression would enhance $S$. aureus colonization. We determined nasal carrier state and long-term hair cortisol levels in 72 healthy subjects. Nasal swabs were collected twice with an interval of 2 weeks. Cortisol levels were determined in hair segments of $3 \mathrm{~cm}$, which corresponds to a period of roughly 3 months. Of all 72 participants, 38 were non-carriers, 10 were intermittent carriers, and 24 were persistent carriers of $S$. aureus. Cortisol levels did not differ between these carrier groups $(p=0.638)$. Long-term cortisol levels are not associated with $S$. aureus nasal carriage.
\end{abstract}

Laura Manenschijn and Andrea M. Jetten contributed equally to this manuscript

L. Manenschijn $(\varangle) \cdot$ A. M. Jetten $\cdot$ J. W. Koper

E. L. T. van den Akker • E. F. C. van Rossum

Department of Internal Medicine, Erasmus Medical Center,

Room Ee-542, PO box 2040, 3000 CA Rotterdam,

The Netherlands

e-mail: 1.manenschijn@erasmusmc.nl

W. J. B. van Wamel · M. Tavakol · A. van Belkum

Department of Medical Microbiology and Infectious Diseases,

Erasmus Medical Center,

Rotterdam, The Netherlands

Present Address:

A. van Belkum

BioMerieux, Microbiology R\&D,

La Balme-les-Grottes, France

\section{Introduction}

Staphylococcus aureus (S. aureus) can colonize the skin and mucosae of humans and is most frequently located in the anterior nares [1]. Carriage of this pathogen is a major risk in the development of various $S$. aureus infections [2]. Worldwide, the prevalence of meticillin-resistant strains is increasing (MRSA). Therefore, it is important to know why some people are more vulnerable to $S$. aureus colonization and what determines the carrier state. Historically, three patterns of carriage are recognized in humans: persistent carriage, intermittent carriage, and no carriage. Persistent carriers have a higher risk of developing $S$. aureus infections and the density of the colonization is higher compared with intermittent carriers [3]. Furthermore, persistent carriers are usually colonized by one specific strain, while intermittent carriers may switch strains over time [4]. However, recently we found that intermittent and non-carriers share similar $S$. aureus nasal elimination kinetics and antistaphylococcal antibody profiles, suggesting that there might be only two types of nasal carriers, namely persistent carriers and others [5].

The pathogenesis of the nasal colonization of $S$. aureus is not completely understood. Host characteristics of the immune system seem to play a role in determining carrier status, as well as environmental and humoral factors in nasal secretions [2]. Another factor that seems to influence carrier status is glucocorticoid (GC) sensitivity. Polymorphisms of the glucocorticoid receptor gene, which are associated with differences in GC sensitivity, have been associated with the $S$. aureus carrier state [6]. Homozygous carriers of the $9 \beta$ polymorphism (rs6198), which is associated with a relative GC resistance with respect to transrepressional effects, had a $68 \%$ decreased risk of persistent nasal carriage. In contrast, subjects with an ER22/23EK+9ß (rs6189+rs6190 and rs6198) polymor- 
phism, which is also associated with a relative resistance, in particular with respect to the negative feedback mechanism and in various tissues, showed an $80 \%$ increased risk of being persistent carriers. The main glucocorticoid in humans is cortisol. Since cortisol is capable of suppressing the immune system and polymorphisms affecting GC sensitivity seem to influence the carriage state of $S$. aureus, long-term cortisol levels may differ between the different carrier states.

In this study we determined cortisol levels in scalp hair to measure long-term cortisol levels. Measurement of cortisol in scalp hair is a recently developed method of measuring long-term cortisol levels [7-11]. The important advantage of this type of cortisol measurement is that there are no limitations caused by the pulsatile secretion of cortisol, the circadian rhythm of cortisol secretion and the variability due to acute circumstances (e.g. physical or emotional stress). Hair grows at approximately $1 \mathrm{~cm}$ per month [12]; therefore, $1 \mathrm{~cm}$ of hair represents the cortisol level in 1 month. Previous studies showed that endogenous cortisol can be measured reliably in scalp hair [7-11]. We hypothesize that higher cortisol levels in hair are associated with persistent nasal carriage of $S$. aureus.

\section{Materials and methods}

Study population

Eighty healthy individuals participated in this study. All subjects filled out a questionnaire regarding gender, age, the use of hair products, hair treatment (perm, coloring, bleaching), and medical status (autoimmune disease and glucocorticoid use). Participants were excluded if they had used glucocorticoids during the last 3 months or had an autoimmune disease. The study was approved by the Medical Ethics committee of the Erasmus Medical Center and all participants gave informed consent.

Nasal carriage of $S$. aureus

Nasal swabs were collected twice at an interval of 2 weeks and were analyzed as previously described [3]. Subjects were defined as persistent carriers when both cultures were positive, intermittent carriers when one of the cultures was positive and non-carriers when none of the cultures was positive for $S$. aureus.

Hair collection and preparation

From all participants, approximately 100 hairs were cut from the posterior vertex. The $3 \mathrm{~cm}$ proximal to the scalp was used for analysis. Since hair grows at an average rate of $1 \mathrm{~cm}$ per month, this corresponds roughly to cortisol levels in the last 3 months. A detailed description of hair preparation has been given by Sauvé et al. [9]. In brief, the 3-cm hair segment was weighted and cut into small pieces in a glass vial with surgical scissors. One milliliter of methanol was added and the vial was incubated for $16 \mathrm{~h}$ overnight at $52^{\circ} \mathrm{C}$, while gently shaking. The methanol was then transferred into a glass tube and evaporated under nitrogen. When dry, the samples were dissolved in $250 \mu \mathrm{L}$ phosphate buffered saline ( $\mathrm{pH} 8.0)$ and vortexed for $1 \mathrm{~min}$ followed by a 30 -s vortex before analysis.

Hair analysis

Cortisol levels were measured using a commercially available ELISA Kit for Salivary Cortisol (DRG International, USA) as per the manufacturer's directions with the reagents provided. Cross reactivity of other steroids with the kit's antibodies was reported as follows: corticosterone $(29.00 \%)$, cortisone (3.00\%), 11-deoxycortisol $(<1.00 \%), 17-\mathrm{OH}$ progesterone $(<0.50 \%)$, and other hormones $(<0.10 \%)$. Intra-assay variation was below $5 \%$ and the inter-assay variation below $8 \%$, as stated by the manufacturer.

Statistical analysis

Statistical analyses were performed using SPSS 17.0 for Windows (SPSS, Chicago, IL, USA). Differences in baseline characteristics between the different carrier state groups were tested using Chi-squared tests and ANOVA. Cortisol levels were log transformed to obtain a normal distribution. ANOVA was used to test the effects of gender, hair treatment, and the use of hair products on cortisol levels. With an ANCOVA the relationship between cortisol levels and the $S$. aureus carrier state was tested, while controlling for gender, age, and hair treatment.

\section{Results}

After exclusion of subjects who used glucocorticoids in the last 3 months or who had an autoimmune disease, 72 individuals were analyzed. Carrier group characteristics are presented in Table 1. There were no significant differences in group characteristics.

After log transformation, hair cortisol levels were normally distributed (Kolmogorov-Smirnov $p=0.200$ ). Hair cortisol levels were significantly lower in the hair treatment group compared with the untreated hair group (23.77 $\mathrm{pg} / \mathrm{mg}$ hair versus $33.42 \mathrm{pg} / \mathrm{mg}$ hair, $\mathrm{F}_{(1,70)}=8.295$, $p=0.005)$. No significant effects of gender $\mathrm{F}_{(1,70)}=2.428$, $p=0.124)$ or use of hair product $\left(\mathrm{F}_{(1,69)}=0.106, p=0.746\right)$ on cortisol levels was found. There was no correlation between age and hair cortisol $\left(r_{\mathrm{s}}=-0.028, p=0.816\right)$. 
Table 1 Group characteristics

\begin{tabular}{|c|c|c|c|c|}
\hline & Non-carriers, $n=38$ & Intermittent carriers, $n=10$ & Persistent carriers, $n=24$ & $p$ value \\
\hline Age, years (range) & $35.42(21-60)$ & $41.00(22-58)$ & $33.71(19-55)$ & 0.272 \\
\hline Gender, women (\%) & $24(63.2)$ & $6(60)$ & $13(54.2)$ & 0.781 \\
\hline Hair treatment $(\%)$ & $8(21.1)$ & $3(30)$ & $8(33.3)$ & 0.543 \\
\hline Use of hair products (\%) & $17(44.7)$ & $8(80)$ & $8(34.8)$ & 0.054 \\
\hline
\end{tabular}

Data are shown as number (\%). Age is shown as mean (minimum to maximum). Hair treatment includes dyeing, bleaching and permanent waving. Hair products used were spray, mousse, gel, and wax

The mean cortisol levels of the carrier groups are shown in Fig. 1. Hair cortisol levels did not significantly differ in the last 3 months between carrier groups $\left(\mathrm{F}_{(2,66)}=0.425, p=0.638\right.$, corrected for hair treatment, age, and gender). In addition, cortisol levels in the combined group of non-carriers and intermittent carriers were not significantly different from cortisol levels in persistent carriers $\left(\mathrm{F}_{(1,67)}=0.837, p=0.363\right)$.

\section{Discussion}

Our study shows that there are no differences in long-term cortisol levels, as measured in scalp hair, among non-carriers, intermittent carriers, and persistent carriers of $S$. aureus. To our knowledge, this is the first study that determined hair cortisol levels with regard to the nasal carriage status of $S$. aureus. Measurement of cortisol in scalp hair is a recently developed method of measuring long-term cortisol levels and seems a reliable measure of long-term cortisol exposure, as shown in several other studies [7-11]. Higher cortisol levels during a longer period of time could lead to an immunosuppressive effect and subsequently to increased $S$. aureus colonization. Theoretically, higher cortisol levels in scalp hair could be associated with persistent nasal carriage of $S$. aureus. However, in the present study we did not observe chronically higher cortisol levels in persistent nasal $S$. aureus carriers.

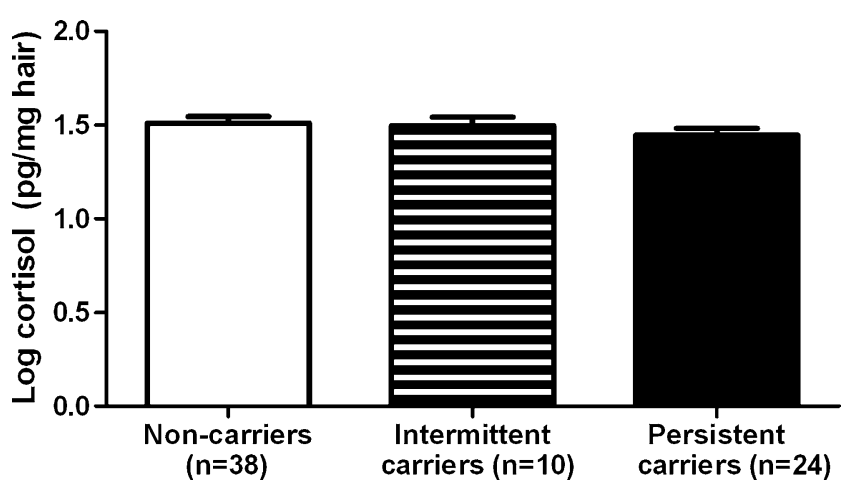

Fig. 1 Mean cortisol levels in the carrier groups. Error bars represent SEM
Interestingly, polymorphisms in the glucocorticoid receptor gene seemed to influence the carrier state of $S$. aureus. We previously showed that the $9 \beta$ polymorphism was associated with a $68 \%$ decreased risk of $S$. aureus carriage [6]. This polymorphism is thought to increase the stability of the GR- $\beta$ isoform, which is a dominant negative inhibitor of the active GR- $\alpha$, and therefore causes a relative GC resistance. This relative resistance could lead to decreased suppression of the immune system by GCs, which would result in a more active immune system, yielding a decreased risk of $S$. aureus colonization. This was indeed the main finding of that study. Furthermore, the ER22/23EK polymorphism of the GR gene was found to be associated with an increased risk of $S$. aureus carriage. The ER22/23EK polymorphism is associated with a relative resistance for GCs for its transactivating effect, but this polymorphism may not affect the immune system since normal transinhibition of the proinflammatory nuclear factor NF- $\mathrm{KB}$ was observed [13]. Although numerous effects throughout the body have been reported for all these GR polymorphisms, no differences in serum cortisol levels have been described [14], suggesting that the mildly altered GC sensitivity is mostly present at the tissue level with a potential cell-specific effect. Since GR polymorphisms only slightly affect GC sensitivity with respect to the negative feedback mechanism, no evident differences in cortisol levels may be observed. This would explain why we did not find any differences in long-term cortisol levels between carrier states despite the fact that there was a clear effect of GR polymorphisms. In our study population, GR polymorphisms were not determined; therefore, an interaction between long-term cortisol levels and GR polymorphisms could not be studied.

Since cortisol levels seem not to significantly influence the risk of $S$. aureus colonization, it is possible that genetics and other host factors are more important. This is also supported by the finding that non-carriers, when inoculated with different $S$. aureus strains, quickly become noncarriers again, while persistent carriers select the strain they were carrying before [15]. These findings suggest that individuals are quite stable regarding their carrier state. 
Cortisol levels can change over time because of emotional or physical stress and are therefore not necessarily stable and possibly a less important host factor for $S$. aureus colonization. Nevertheless, we cannot rule out that cortisol may indirectly play a role in $S$. aureus colonization by interacting with host factors of the immune system and an individual's genetic make-up. Interestingly, persistent $S$. aureus colonization does not seem to lead to greater physical stress since cortisol levels would then be increased in persistent carriers.

In conclusion, we found no differences in long-term cortisol levels, measured in scalp hair, among non-carriers, intermittent carriers, and persistent carriers of $S$. aureus in the anterior nares. Thus, cortisol levels seem not to significantly affect the carriage state of $S$. aureus.

Acknowledgements This work was supported by a grant from The Netherlands Organisation for Scientific Research (NWO; grant number 916.96.069). No writing assistance was provided in the preparation of this manuscript.

Conflict of interest The authors declare no conflict of interest.

Open Access This article is distributed under the terms of the Creative Commons Attribution Noncommercial License which permits any noncommercial use, distribution, and reproduction in any medium, provided the original author(s) and source are credited.

\section{References}

1. Williams RE (1963) Healthy carriage of Staphylococcus aureus: its prevalence and importance. Bacteriol Rev 27:56-71

2. Wertheim HF, Vos MC, Ott A, van Belkum A, Voss A, Kluytmans JA, van Keulen PH, Vandenbroucke-Grauls CM, Meester MH, Verbrugh HA (2004) Risk and outcome of nosocomial Staphylococcus aureus bacteraemia in nasal carriers versus non-carriers. Lancet 364(9435):703-705

3. Nouwen JL, Ott A, Kluytmans-Vandenbergh MF, Boelens HA, Hofman A, van Belkum A, Verbrugh HA (2004) Predicting the
Staphylococcus aureus nasal carrier state: derivation and validation of a "culture rule". Clin Infect Dis 39(6):806-811

4. Eriksen NH, Espersen F, Rosdahl VT, Jensen K (1995) Carriage of Staphylococcus aureus among 104 healthy persons during a 19-month period. Epidemiol Infect 115(1):51-60

5. Van Belkum A, Verkaik NJ, de Vogel CP, Boelens HA, Verveer J, Nouwen JL, Verbrugh HA, Wertheim HF (2009) Reclassification of Staphylococcus aureus nasal carriage types. J Infect Dis 199 (12):1820-1826

6. Van den Akker EL, Nouwen JL, Melles DC, van Rossum EF, Koper JW, Uitterlinden AG, Hofman A, Verbrugh HA, Pols HA, Lamberts SW, van Belkum A (2006) Staphylococcus aureus nasal carriage is associated with glucocorticoid receptor gene polymorphisms. J Infect Dis 194(6):814-818

7. Kalra S, Einarson A, Karaskov T, Van Uum S, Koren G (2007) The relationship between stress and hair cortisol in healthy pregnant women. Clin Invest Med 30(2):E103-E107

8. Pereg D, Gow R, Mosseri M, Lishner M, Rieder M, Van Uum S, Koren G (2011) Hair cortisol and the risk for acute myocardial infarction in adult men. Stress 14(1):73-81

9. Sauvé B, Koren G, Walsh G, Tokmakejian S, Van Uum SH (2007) Measurement of cortisol in human hair as a biomarker of systemic exposure. Clin Invest Med 30(5):E183-E191

10. Stalder T, Kirschbaum C, Heinze K, Steudte S, Foley P, Tietze A, Dettenborn L (2010) Use of hair cortisol analysis to detect hypercortisolism during active drinking phases in alcoholdependent individuals. Biol Psychol 85(3):357-360

11. Van Uum SH, Sauvé B, Fraser LA, Morley-Forster P, Paul TL, Koren G (2008) Elevated content of cortisol in hair of patients with severe chronic pain: a novel biomarker for stress. Stress 11 (6):483-488

12. Pragst F, Balikova MA (2006) State of the art in hair analysis for detection of drug and alcohol abuse. Clin Chim Acta 370 (1-2):17-49

13. Russcher H, van Rossum EF, de Jong FH, Brinkmann AO, Lamberts SW, Koper JW (2005) Increased expression of the glucocorticoid receptor-A translational isoform as a result of the ER22/23EK polymorphism. Mol Endocrinol 19(7):16871696

14. Manenschijn L, van den Akker EL, Lamberts SW, van Rossum EF (2009) Clinical features associated with glucocorticoid receptor polymorphisms. An overview. Ann NY Acad Sci 1179:179-198

15. Nouwen J, Boelens H, van Belkum A, Verbrugh H (2004) Human factor in Staphylococcus aureus nasal carriage. Infect Immun 72 (11):6685-6688 